\title{
Crise de la société, crise de l'école
}

Le cas du Cameroun

Crisis in Society, Crisis in Schooling: The Case of Cameroon

Crisis de la sociedad, crisis de la escuela: el caso de Camerún

\section{Pierre Marie Njiale}

\section{OpenEdition \\ Journals}

Édition électronique

URL : http://journals.openedition.org/ries/1151

DOI : $10.4000 /$ ries. 1151

ISSN : 2261-4265

\section{Éditeur}

Centre international d'études pédagogiques

\section{Édition imprimée}

Date de publication : 1 avril 2006

Pagination : 53-63

ISSN : 1254-4590

\section{Référence électronique}

Pierre Marie Njiale, «Crise de la société, crise de l'école », Revue internationale d'éducation de Sèvres [En ligne], 41 | avril 2006, mis en ligne le 23 novembre 2011, consulté le 08 janvier 2020. URL : http:// journals.openedition.org/ries/1151; DOI : 10.4000/ries.1151 


\section{Crise de la société, crise de l'école}

\section{Le cas du Cameroun}

\section{Pierre Marie Njiale}

Il y a un moment où un peuple cherche à savoir d'où il vient et où il va. Interroger l'école primaire au Cameroun, c'est aussi questionner l'histoire, le présent et anticiper le futur. On sait que tout système éducatif est à l'image de la société qui a la charge de l'instituer et de l'organiser. On ne peut en dire autant des sociétés qui ont vécu l'expérience de la domination. Ici, l'éducation n'est pas l'aboutissement d'un développement interne car l'école a constitué un leitmotiv de la politique coloniale. Toutefois, c'est sous le signe de la renaissance et de l'espérance que l'éducation primaire prend son envol au lendemain des indépendances. À ce titre, son lent cheminement est plein d'enseignements.

\section{RAPPEL HISTORIQUE La scolarisation allemande}

Les débuts de la scolarisation au Cameroun sont précis et bien connus. En effet, l'école trouve ses marques avec le pasteur Baptiste Joseph Merrick, qui arrivé à Bimbia en 1883, ouvre l'année suivante (1884) une école. Merrick bénéficie de l'aide précieuse du pasteur anglais Alfred Saker. Plus tard, les baptistes sont rejoints par d'autres congrégations missionnaires bâlois d'obédience presbytérienne. Toutes oeuvrent dans le domaine scolaire et religieux. En dépit de leur âge avancé, les enfants intègrent directement les écoles ouvertes par les missionnaires. En dehors de l'évangile, les élèves y apprennent en anglais et en langues locales quelques rudiments de lecture, d'écriture et de chant.

Le 12 juillet 1884, le Cameroun devient un protectorat allemand. L'administration présente dans le territoire ne possède pas une véritable politique scolaire. Les missionnaires ont le monopole de l'enseignement qui a un caractère essentiellement "primaire» et vise à former des auxiliaires pour l'administration et le secteur privé naissant. À la veille des hostilités qui entraînent de facto le départ des Allemands en 1914, les écoles publiques et privées comptent un effectif de 36823 élèves.

\section{La scolarisation française et britannique}

L'issue de la guerre (1914-1916) a pour effet, l'éclatement du Cameroun allemand en deux territoires. La France s'adjuge le Cameroun oriental, l'Angleterre hérite du Cameroun occidental. L'administration de ces territoires 
dure le temps du mandat (1920-1944) et de la tutelle (1945-1959). En matière d'éducation, les deux puissances n'adoptent pas la même politique. Pour ce qui est du territoire sous administration française, l'œuvre scolaire commencée par les Allemands est poursuivie. On y associe fort heureusement les missionnaires. Sous le mandat, un arrêté du 25 juillet 1921 institue un enseignement primaire et élémentaire qui est dispensé dans les écoles de brousse ou de villages, celles-ci étant coiffées par les écoles urbaines et régionales. Les études culminent à l'école primaire supérieure où sont formés les grands commis de l'administration. Au Cameroun anglophone, l'enseignement primaire est exclusivement confié aux missions chrétiennes dans le cadre de l'Approved Volontary Agency.

En 1945, on développe au Cameroun les mêmes cycles d'enseignement qu'en métropole. Le certificat d'études primaire sanctionne le premier degré.

Au moment où la France quitte le territoire en 1959, l'enseignement primaire connaît un niveau très avancé. Il affiche un taux de scolarisation de $45 \%$. On se trouve là en présence d'un acquis qu'il faut conserver et améliorer.

\section{NAISSANCE ET ÉVOLUTION D'UN ENSEIGNEMENT PRIMAIRE NATIONAL}

La période 1960-1972 constitue une période fondatrice. Le développement de la scolarisation s'inscrit dans la continuité de la tradition héritée de l'époque coloniale. Le premier gouvernement du Cameroun indépendant dispose d'un secrétariat d'État à l'enseignement primaire. L'éducation préscolaire est inexistante. L'école primaire constitue alors le premier degré de l'apprentissage scolaire. Conscients de ce que l'éducation peut apporter au développement humain et social, les nouveaux responsables y consacrent une part importante du budget de l'État. L'attention accordée à l'éducation s’intègre dans une démarche globale de transformation de la société. L'année 1961 consacre la République fédérale et la réunification du Cameroun francophone et anglophone. Chacun des gouvernements des deux États possède un secrétariat d'État à l'enseignement primaire. L'éducation au niveau fédéral est coordonnée par un ministère en charge de l'éducation.

La loi fédérale $n^{\circ}$ 63-13 du 19 juin 1963, qui préfigure les structures actuelles de l'éducation, réglemente l'enseignement primaire et secondaire. Au niveau primaire, il est prévu deux cycles parallèles de six ans pour les francophones et sept ans pour les anglophones. Cette organisation s'inspire des expériences passées vécues dans chaque État. Elle reflète par ailleurs la volonté affichée de préserver l'unité nationale encore fragile et le souci de consolider la spécificité de chaque entité linguistique. On le voit, l'unification structurelle en vue d'un enseignement primaire identique pour tous les enfants ne semble pas prioritaire. Le français et l'anglais sont adoptés comme langues d'enseignement. 
L'orientation politique du premier plan quinquennal de développement économique social et culturel (1961-1966) affirme la priorité donnée à l'éducation. Les objectifs du plan préconisent, entre autres, l'adaptation de l'enseignement primaire aux réalités nationales et aux besoins économiques du pays. À cette fin, l'option pratique retenue est la ruralisation de l'école. Elle s'entend comme un souci de former une main-d'œuvre rentable et des élèves attachés à leur milieu par l'apprentissage d'un métier. De fait, le fond de la ruralisation est de promouvoir, par le biais de l'école primaire, une économie rurale. Le principe de base repose sur l'idée que l'école doit retenir à la terre les jeunes instruits.

Le second plan quinquennal (1966-1971) réaffirme l'engagement des responsables à faire de l'éducation un facteur de développement et de croissance. Dans cette perspective, le plan envisage l'accroissement des structures de formations primaires afin de rapprocher les écoles des zones rurales. Le secteur privé confessionnel et laïque est associé à cet effort. À tous égards, l'école primaire enregistre un progrès considérable. C'est la période de l'expansion scolaire. Le Cameroun connaît alors un taux de scolarisation primaire élevé (65\%) qui le place parmi les pays les mieux lotis d'Afrique francophone. Le niveau primaire répertorie 18115 salles de classes en 1970-1971, 17942 enseignants pour un effectif de 815127 élèves $^{1}$. Les résultats aux examens officiels, certificat d'études primaires et élémentaires (CEPE), entrée en $6^{\mathrm{e}}$, sont significatifs d'un rendement interne satisfaisant. La forte demande sociale d'éducation observée en ce temps, ainsi que l'attestent les données disponibles, ne tient pas à la seule poussée démographique. Elle est aussi le fruit d'une situation économique en croissance. À ces raisons s'ajoute le fait que, ici, l'école représente la modernité et est appréciée des populations. Elle représente pour elles un outil formidable d'ascension professionnelle et sociale. Le CEPE, qui sanctionne les études du premier degré, a la valeur d'un «passeport pour l'emploi ». Le système néanmoins n'est pas exempt de difficultés et de disparités. Dans les zones rurales, on note un déficit d'écoles et d'instituteurs. Les classes dans ces localités sont surchargées, un maître pouvant tenir deux à trois classes à la fois. La question semble préoccuper les autorités et sera prise en compte dans les plans quinquennaux ultérieurs.

Durant les années 1972-1984, de grands changements s'opèrent. La République unie succède à la République fédérale. Le législateur scolaire définit les différents ordres d'enseignement (primaire, secondaire et supérieur). Une des directions du ministère de l'éducation nationale a la charge de l'enseignement maternel et primaire. Le troisième plan quinquennal (1971-1976), ainsi que le quatrième (1976-1981) et le cinquième (1981-1986), témoignent des efforts et des ambitions à faire de l'éducation la clef de voûte du développement.

1. Rapport sur le développement humain au Cameroun, PNUD, 1993. 
Le cap de la ruralisation est maintenu, voire renforcé. À Yaoundé (1975) et à Buéa (1977) sont créés des instituts pédagogiques à vocation rurale (IPAR) destinés à apporter un soutien académique à l'enseignement primaire.

Grâce aux subventions accordées, le secteur privé prend une part active à la scolarisation maternelle et primaire. Au tournant des années quatre-vingts, l'accroissement des effectifs se poursuit de façon soutenue. Le taux de scolarisation atteint $75 \%$ en 1985. La croissance scolaire, cependant, n'est pas homogène dans toutes les provinces du pays. Celles du nord et de l'extrême-nord présentent de nombreuses insuffisances. En matière d'encadrement par exemple, elles enregistrent un ratio élève-maître au dessus de 60, voire 100, alors qu'il est de 45 dans les provinces du Sud-Cameroun. Dans l'ensemble, le panorama que présente l'école primaire est de qualité relativement acceptable.

La crise macro-économique à laquelle le Cameroun est confronté entre 1986-2000 freine la croissance et entraîne par ricochet le recul du niveau de scolarisation primaire. Il en résulte que les ressources financières courantes d'éducation par enfants de six à quinze ans sont divisées par deux ${ }^{2}$. Les taux de fréquentation scolaire font apparaître un phénomène de déscolarisation. Les différents diagnostics posés par les organisations internationales (UNESCO, Banque mondiale), dans le cadre de l'ajustement structurel auquel est soumis le pays, établissent le constat d'un niveau scolaire en dysfonctionnement en raison de nombreuses carences liées au faible rendement interne et à l'absence d'objectifs stratégiques de formation. C'est dans ce contexte que le Cameroun souscrit à l'objectif mondial de "l'éducation pour tous » exprimé lors de la conférence sur l'éducation organisée à Jomtien (Thaïlande) en 1990. Dans la foulée se tiennent en 1995 des états généraux de l'éducation. Un constat sévère de l'état de l'éducation primaire est établi. Il en ressort de nombreuses recommandations allant dans le sens de la réforme de l'école de base et du système éducatif en général. Pour éviter la dégradation de la situation, on engage quelques tentatives de réformes. La constitution de 1996 instaure la scolarité primaire obligatoire. Des mesures subséquentes prescrivent en 1997 la gratuité de l'école primaire tout au moins dans les établissements publics (1997). Il faut sans doute voir dans la création récente (2004) d'un ministère de l'éducation de base une tendance volontariste à trouver des solutions aux nombreux problèmes auxquels fait face à présent l'enseignement maternel et primaire.

\section{LA SCOLARISATION MATERNELLE ET PRIMAIRE}

L'équilibre d'un système éducatif repose sur l'agencement de plusieurs dimensions qui intègrent les domaines cognitif, psychomoteur, affectif en relation

2. Gacougnolle et Mingat. Zoom sur un système éducatif. Le Cameroun, 2004. 
avec l'environnement sociologique et politique. Les indicateurs essentiels ici retenus pour l'analyse interne du dispositif de l'enseignement primaire devraient apporter un éclairage utile sur le système actuel. Ceux-ci sont : l'âge et la durée de scolarisation, les missions et objectifs poursuivis, la scolarisation des filles, les programmes de formation, l'évaluation des acquis et la pertinence du système.

\section{Durée incertaine de la scolarisation obligatoire}

Jusqu'en 1971, l'enseignement préscolaire n'est pas un passage obligatoire de la scolarisation. L'éducation des moins de six ans reste cependant une préoccupation du législateur. Sans faire l'objet d'un texte organique comme le primaire, l'enseignement préscolaire est assuré dans trois sortes d'établissements : les jardins d'enfants, placés sous la responsabilité du ministère de la santé ; les classes enfantines et les écoles maternelles placées sous l'autorité du secrétariat d'État à l'enseignement primaire. Toutes ces structures sont des « classes gardiennes » ouvertes aux enfants âgés de trois à cinq ans qui attendent l'âge de six ans pour être admis à la section d'initiation au langage (SIL).

Dans les structures actuelles de l'éducation, l'enseignement maternel et primaire s'adresse aux enfants âgés de trois à treize ans. La maternelle recrute des tout petits de deux à six ans. Ils sont initiés pendant trois ans à l'apprentissage de la langue (le français ou l'anglais) et de l'écriture. Pour l'essentiel, c'est l'école de la socialisation. On y apprend, en outre, les bonnes habitudes, des jeux éducatifs. L'accent est mis sur l'épanouissement total de l'enfant. Dans le réseau francophone, l'enseignement maternel comprend trois classes : la petite, la moyenne et la grande section. La «nursery school» dans le réseau anglophone comporte deux classes : class 1 et class 2. Passé ce stade dans les deux sous-systèmes, l'enfant accède au cycle primaire.

L'enseignement primaire, indépendamment du sous-système, dure six ans. Il conduit en fin de cycle à l'obtention du certificat d'études primaires (CEP) qui équivaut au Level Certificate $(O L C)$ du système anglophone, et permet de présenter le concours d'entrée en $6^{\mathrm{e}}$ du secondaire.

En rendant l'enseignement primaire obligatoire, l'État en a fait l'une des grandes priorités nationales, mais il est essentiel de relever que cet objectif s'inscrit plus dans un ordre politique que dans la réalité. Les textes, en effet, ne disent pas à quel âge l'obligation scolaire commence et se termine. De même, aucune mesure n'a été prise pour rendre effectif ce principe. L'enseignement primaire demeure payant dans les établissements privés qui accueillent près de $45 \%$ des effectifs. La gratuité scolaire ne saurait s'imposer si les familles ne sont pas exemptes de tous les frais. Pour un sujet si important où il y va de l'avenir de la jeunesse, on ne peut se contenter des seules déclarations d'intention des responsables. Une réflexion profonde sur cette question paraît indispensable. 


\section{FINALITÉS ET MISSIONS DE L'ÉCOLE PRIMAIRE}

Tout projet éducatif renvoie à une conception philosophique et à un système de valeurs en rapport avec la société qu'on entend édifier et le type d'homme qu'on souhaite développer. La constitution de la République de 1996 éclaire fort à propos sur les fondements sociologiques et politiques de l'enseignement primaire. Elle affirme en son préambule « le devoir impérieux de l'État d'assurer à l'enfant le droit à l'instruction, d'organiser et de contrôler l'enseignement à tous les degrés ». Elle stipule par ailleurs l'obligation de la scolarisation primaire.

Partant de ces principes, l'école primaire, l'école de base, épouse une vision qui lui assigne plusieurs finalités. La toute première est de faire acquérir à l'enfant des connaissances de base (lire, écrire, compter, analyser et agir sur son environnement) en vue de son développement intellectuel. Véritable école d'initiation, elle propose en second lieu l'acquisition de comportements tels que l'amour de la patrie, l'amour du travail bien fait, la culture de l'effort et du progrès, le sens des responsabilités, le sens du civisme et de la discipline. Elle poursuit enfin l'acquisition de savoir-faire. Pour une éducation intégrale et adaptée de l'enfant, l'école primaire se voit ainsi confier deux autres missions : le renforcement de l'unité et de la cohésion nationales; le développement du bilinguisme par l'initiation de l'enfant à la seconde langue nationale.

S'il est vrai que ces objectifs sont fondamentaux et patriotiques, il n'en demeure pas moins que leurs réalisations concrètes sont difficiles du fait qu'on observe de nombreux écarts entre l'intention affichée et les applications sur le terrain. Le bilinguisme, pour s'en tenir à cet exemple, ne semble pas être une réalité vivante dans les deux sous-systèmes francophone et anglophone du primaire.

En s'intéressant à l'éducation à la citoyenneté, qui est l'un des axes forts des objectifs récents de l'éducation, on constate que les enseignements afférents sont réduits à la portion congrue et se limitent aux leçons de morale, à l'étude du code de la route, aux rudiments de l'hygiène sans que l'accent soit mis sur l'esprit civique, patriotique ou que le contenu fasse allusion à la bonne gouvernance.

\section{La scolarisation des filles}

En développant une éducation primaire mixte dès le début, le législateur scolaire semble avoir mesuré ici les enjeux d'une scolarisation féminine. Les textes préconisant l'égalité des sexes devant l'instruction et garantissant à tous le droit à l'éducation, la scolarisation des filles s'est développée en s'intégrant à la dynamique d'ensemble de l'enseignement. Elle ne fait pas l'objet d'une disposition particulière mais constitue une préoccupation des pouvoirs publics. En 
tout état de cause, on peut dire que la présence féminine dans le primaire, peu significative au départ, a connu une évolution remarquable pour atteindre, ces dix dernières années, un taux moyen comparable à celui des garçons. Les statistiques officielles $^{3}$ affichent en 2004 au niveau primaire un effectif de 1329106 filles contre 1577626 garçons. Selon les recherches ${ }^{4}$, les filles réussissent dans leurs études autant, sinon mieux, que leurs condisciples masculins. Il se dégage des résultats aux examens du CEPE (2004), qu'elles obtiennent un taux de réussite appréciable, comparable à ceux des garçons (48\% et $52 \%)$. On estime que, pour les années à venir, le nombre de filles va continuer de s'accroître d'autant que pour une classe d'âge, les filles ont un taux de survie scolaire supérieure à celui des garçons. En ce domaine, les données font cependant apparaître des disparités, et notamment un faible taux de scolarisation des filles dans les provinces septentrionale et de l'est. Cette situation s'explique par des motifs liés aux us et coutumes, à la religion, au mariage précoce et au travail des jeunes filles, celles-ci étant, dans ces localités, l'objet de sollicitations parentales plus exigeantes que leurs frères. Jusqu'en 1995, seules $35 \%$ des femmes, au niveau de l'ensemble de la population active, avaient dépassé le niveau primaire $^{5}$. À la lumière de ce constat, on a toutes les raisons de penser que beaucoup reste à faire dans la réflexion pour trouver les voies et moyens pouvant améliorer l'accès des filles et des groupes défavorisés à l'éducation fondamentale. L'enseignement de base représente un élément clé du dispositif de formation. Élargir son accès à toutes les catégories sociales serait un moyen efficace de lutter contre l'analphabétisme et de promouvoir l'équité.

\section{Programmes encyclopédiques et pratiques pédagogiques ambitieuses}

Les programmes en vigueur dans les écoles publiques et privées sont du ressort du ministère de l'éducation nationale qui a la charge de les développer. Ils ont un caractère national et conduisent à des examens permettant la délivrance des diplômes nationaux (CEP, OLC) ou à l'accès par concours en $6^{\mathrm{e}}$ ou en form $1 \mathrm{du}$ secondaire anglophone.

Les programmes, par leur volume et leur contenu, ont un caractère encyclopédique. Sans être organisés sous forme de curricula, ils présentent de façon détaillée les objectifs à atteindre et les contenus devant être dispensés aux élèves. De façon concrète, ils visent une formation générale et l'absence d'outils documentaires, technologiques et de moyens didactiques constitue l'une de leurs faiblesses. Les textes en la matière recommandent à l'instituteur l'adaptation

\footnotetext{
3. Carte scolaire de l'éducation au Cameroun, MINEDUC, 2004.

4. Gacougnolle et Mingat, 2004.

5. La formation et l'emploi au Cameroun, OIT/MTPS, 1995.
} 
des leçons au développement psychologique de l'enfant et aux réalités sociales et culturelles du milieu. L'enseignant est invité à cet effet à faire usage des exemples familiers à l'enfant.

De la SIL au CM2, les programmes ménagent une place considérable à l'expression française et anglaise, au calcul et aux exercices d'observation. L'éducation civique, le chant, le dessin, l'histoire, la géographie, la biologie, le travail manuel et l'éducation physique et sportive sont dispensés au même titre que les autres matières du programme.

Au Cameroun, les instructions officielles imposent aux enfants du primaire une répartition scolaire annuelle de neuf mois (trente-six semaines) subdivisée en trois trimestres. Les enseignements hebdomadaires s'étalent sur cinq jours, du lundi à vendredi. Une journée de classe comprend cinq à sept heures de cours. La souplesse des programmes permet une vacance des classes le mercredi après midi.

Sur le plan purement pédagogique, les textes ministériels évoquaient jusqu'en 1995 l'usage, pour les maîtres, de méthodes actives, participatives et attrayantes. Cette pratique a progressivement fait place, depuis 1990, à une pédagogie axée sur "l'approche par compétences ». Cette réforme, qui s’est faite dans le cadre de ce qu'on a appelé la " nouvelle approche pédagogique (NAP)» avait pour ambition de vulgariser auprès des instituteurs une démarche d'intervention centrée sur les objectifs et les compétences à faire acquérir. Faute d'évaluation, on ne saurait apprécier les améliorations que ce dispositif a apportées. Le manque de données relatives aux indicateurs de performances constitue aussi l'une des faiblesses du système.

La pédagogie a changé, mais rien ne semble avoir évolué dans la pratique d'évaluation des productions scolaires. Elle manque de congruence avec un enseignement par objectifs. De façon immuable et dans la logique des instructions, le dispositif d'évaluation préconise, au niveau de chaque classe, des contrôles continus de connaissances et un examen de fin de trimestre. Le passage en classe supérieure est sanctionné par l'obtention d'une note moyenne égale ou supérieure à 10 et traduisant la sommation des performances de l'élève aux trois trimestres de l'année. À la fin de chaque trimestre scolaire, un bulletin d'évaluation met à la disposition des parents les informations sur les performances des enfants. Les évaluations ont une finalité plus sommative que formative. Dans l'état actuel, l'évaluation des travaux scolaires ne semble pas être un outil de gestion et de pilotage du système. De fait, les textes ne prévoient pas une évaluation nationale des apprentissages scolaires des élèves au cours du cycle primaire. Le CEPE et le concours d'entrée en $6^{\mathrm{e}}$ ou au form 1 sont les seuls baromètres de comparaison nationale du niveau de connaissances atteint par les élèves. N'ayant pas été conçus pour cela, ces dispositifs ne sauraient objectivement servir à cette finalité. Néanmoins, les données internationales disponibles permettent d'apprécier le niveau des acquis scolaires. En effet, les enquêtes 
comparatives du PASEC $^{6}$ et du MLA ${ }^{7}$, portant sur plusieurs pays africains, dont le Cameroun, ont montré un faible niveau de performance chez les élèves en ce qui concerne la lecture et le calcul. Les mêmes études révèlent qu'en fin de cycle primaire, la plupart des élèves ne possèdent pas les savoirs et les compétences de vie (life skills) requis.

\section{Le maître}

Les programmes confèrent au maître le rôle d'acteur central de la relation. Il est "principal garant de la qualité de l'éducation»; il est exigé de lui une bonne culture générale, des « qualités physiques, professionnelles, intellectuelles et morales ». De par les responsabilités qui lui incombent, c'est l'homme capable de tout enseigner. Il dispense des leçons d'anglais, de français, doit porter une attention particulière à la lecture, devra éviter le rabâchage... Son action pédagogique ne se limite pas au choix de la méthode, "il doit veiller à la progression de l'enfant... ». Les instructions l'invitent à s'en tenir à « ce qu'il n'est pas permis d'ignorer" tout en respectant les principes de la psychologie notamment pour ce qui est de l'âge de l'enfant, de ses aptitudes et de son milieu socioculturel. À l'image de l'écolier qui doit "obéissance ", le maître a l'obligation d'enseignement, d'éducation, d'encadrement pédagogique et d'évaluation... $»^{8}$. Au cours de la période d'âge d'or de l'école primaire, de nombreuses écoles normales d'instituteurs (ENI, ENIA) avaient été ouvertes en vue de la formation initiale des maîtres.

Dans les années 80, le rythme avait été d'ouvrir une école normale par province. Le Cameroun comptait en 1990-1991 près d'une vingtaine d'établissements de ce type disséminés sur l'ensemble du territoire. Ils formaient environ 1000 instituteurs par an. La multiplication des ENI tenait à la volonté de disposer d'un corps d'instituteurs qualifiés et en nombre suffisant. Les études estimaient le déficit en ce domaine à près de 18000 maîtres. L'entrée aux ENI par voie de concours ou sur titre était réservée aux titulaires du baccalauréat de l'enseignement secondaire. Les élèves-maîtres percevaient un salaire et à l'issue de deux ans de formation étaient intégrés dans la fonction publique. Il est clair qu'aujourd'hui l'instituteur a perdu du prestige et de l'aura qu'il avait auprès des élèves et des familles. Depuis les années quatre-vingt-dix, la situation des maîtres n'a cessé de se détériorer. Du fait de la mise en place des mesures de l'ajustement structurel, nombre d'enseignants ont été contraints aux « départs volontaires ». Cette politique a aussi conduit au gel de recrutement des instituteurs. De fait, les effets pervers de la crise économique ont considérablement affecté les conditions de travail des maîtres. La baisse des salaires les a contraints à trouver d'autres moyens de survie. Cette situation n'a pas manqué d'affecter

\footnotetext{
6. Programme d'analyse des systèmes éducatifs de la CONFEMEN, 2003.

7. Projet pour l'évaluation des acquis scolaires (MLA).

8. Loi d'orientation de l'éducation, 1998.
} 
la qualité de l'enseignement. Le ratio d'encadrement élève/maître qui était de 49 en 1971-1972 est passé à 82, voire 120 en 2004.

\section{COMMENT ARRÊTER LA DÉGRADATION DE L’ÉCOLE PRIMAIRE CAMEROUNAISE ?}

Il est incontestable que le système s'est dégradé continuellement. Aujourd'hui, l'école primaire au Cameroun, à l'image de la société, offre la physionomie d'une institution en crise traversée par de multiples malaises. Quarante ans après, ce niveau de formation n'a pas tenu ses promesses. Les résultats restent contrastés au regard des investissements. L'ambition affirmée d'un enseignement primaire quantitatif parait certes relativement accomplie, mais que peut valoir la quantité sans la qualité. Dans le contexte actuel, l'école primaire se trouve confrontée à une série de problèmes. Les constats ${ }^{9}$ ont permis d'établir ce qui suit :

- une demande massive de la scolarisation par rapport à une offre insuffisante d'enseignement maternel et primaire ;

- un taux de déperdition élevé ; le taux de redoublement étant estimé à $30 \%$, le taux d'abandon à $15 \%$. Certains élèves mettent dix années pour sortir du cycle primaire ;

- l'absence d'une structure d'évaluation des programmes et des méthodes d'enseignement ;

- des classes surchargées ;

- une poussée des inégalités des chances scolaires et des disparités scolaires régionales;

- des infrastructures et des moyens didactiques insuffisants;

- une crise de recrutement d'enseignants qui ne résulte pas d'un manque de motivation mais qui est le corollaire des disponibilités financières ;

- l'analyse des flux d'élèves montrant que le sous-système anglophone de scolarisation primaire obtient des performances supérieures à celles du soussystème francophone; $59 \%$ de nouveaux élèves dans le système francophone atteignent la sixième année du cycle ${ }^{10}$, contre $80 \%$ dans le système anglophone.

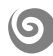

L'efficacité d'un système éducatif réside à juste titre en sa capacité à muer pour s'adapter aux exigences du contexte et des évolutions. Cette approche prospective semble avoir manqué au tournant des années quatre-vingt-dix.

9. États généraux de l'éducation, 1995.

10. Gacougnolle et Mingat, 2004. 
L'état de la situation de l'école primaire conforte l'option d'une réforme structurelle et managériale profonde. Décréter l'école obligatoire ne constitue pas une fin en soi. En donnant la priorité à l'éducation de base, les moyens devraient suivre pour une plus grande couverture scolaire.

L'analyse des résultats obtenus permet de craindre que l'éducation primaire pour tous ne demeure un idéal qui sera loin d'être atteint en 2015. Adhérer à la scolarisation pour tous, c'était faire fi d'ignorer les nombreux obstacles qui pour les pays de ce niveau constituent des goulots d'étranglement. Relever le défi de l'éducation primaire pour tous suppose des politiques éducatives qui tiennent compte des ressources financières insuffisantes et de l'utilisation efficace des faibles moyens disponibles.

L'orientation nouvelle à donner à l'école primaire requiert imagination et inventivité. L'objectif de l'amélioration des performances scolaires et d'une scolarisation de qualité devrait intégrer la possibilité de faire appel aux acteurs de la société civile, du secteur privé et autres partenaires afin de mobiliser toutes les bonnes volontés et les soutiens nécessaires dans une vision partagée.

Une école primaire efficiente impose la mobilisation et une implication effective de ceux qui ont la responsabilité de transmettre le savoir. Les réformes devraient privilégier l'amélioration de la condition enseignante. Pour accroître l'accès à l'éducation de base versus la demande, on devrait renforcer les mesures tendant à la gratuité de l'enseignement et garantissant le droit à l'éducation. Il est convenu que les réformes tant souhaitées sont difficiles à mettre en œuvre vu les contraintes contextuelles et macro-économiques. Mais elles demeurent salutaires et déterminantes pour relever les défis d'une éducation de base pour tous d'ici l'an 2015. Une vision optimale d'un enseignement primaire efficace et démocratique exige que l'on dispose des données pertinentes pour une évaluation juste du dispositif.

\section{BibLIOGRAPHIE}

CAPELLE Jean (1990): L'éducation en Afrique noire à la veille des indépendances, 1946-1958, préface de L.S. Senghor. Paris, Kartala.

GACOUGNOLLE Luc, MINGAT Alain (2004) : "Zoom sur un système éducatif. Le Cameroun », Lettre de l'ADEA, janvier-mars, pp. 21-24.

VLADIMI Sylla (2004): "Assessing the quality of education in Africa ". ADEA News Letter, janvier-mars, pp. 10-12.

La réforme de l'éducation au Cameroun (1978): ONAREST/ISH.

Le système éducatif au Cameroun. Guide du chef de l'établissement. Édition $1995 / 1996$.

MUNGAH Tchombé (1993): L'accès des filles à l'éducation de base et à l'enseignement primaire au Cameroun. Unesco. 
